\title{
DE-OCCULTATION X-RAY EVENTS OF 2 DECEMBER, 1967
}

\author{
HAROLD ZIRIN and WILLIAM INGHAM, \\ Mount Wilson and Palomar Observatories, Carnegie Institution of Washington, \\ California Institute of Technology \\ and \\ HUGH HUDSON and DAVID McKENZIE \\ University of California, San Diego, Calif., U.S.A.
}

(Received 11 April, 1969)

\begin{abstract}
A flare rising from behind the solar limb was recorded simultaneously by the UCSD X-ray detector on OSO-III (7.7-200 keV) and the Caltech photoheliograph on Robinson Laboratory roof $(\mathrm{H} \alpha)$. The de-occultation gives excellent spatial resolution of the X-ray source. Spectra suggest that the material was already heated to $27000000^{\circ}$ and that the increase in flux was due to the de-occultation. The flux rise to maximum was proportional to the apparent area. The uniformity of this rise shows that there was no special kernel of emission. Comparison of the deduced volume with the bremsstrahlung formula gives a density of about $10^{10}$ for the $27000000^{\circ}$ component of the flare; this is confirmed by consideration of the maximum possible coulomb braking. The actual decay is more likely by escape rather than coulomb braking.
\end{abstract}

In the course of studies of soft X-rays observed by the OSO-III X-ray telescope (Hudson et al., 1969) simultaneous with observations of solar phenomena with the Caltech photoheliograph, a particularly interesting de-occultation event occurred on December 2, 1967. A large bright limb flare rose from behind the limb of the sun, coming from a region that had crossed the limb just a few hours earlier. By comparison of the uncovered area of the flare and the observed X-ray flux, it was possible to draw conclusions on the plasma responsible for the X-ray event. This plasma was found to be uniformly distributed through the arch. The electrons had energy corresponding to $27000000^{\circ}$ (lower than the typical flare). The density of these energetic electrons was between 6 and $9 \times 10^{9} \mathrm{~cm}^{-3}$.

\section{Observations}

An active sunspot group crossed the west limb of the sun between December 1 and 2, 1967. Observations with the Caltech photoheliograph (the observers were Bohlin and Zirin) show a number of limb events during the day. These for the most part were small sprays, each of which corresponds quite well with an X-ray event. The times of the events observed by the UCSD X-ray telescope on OSO-III in the 7.7-12.6 keV energy range, as well as the observed solar events, are given in Table I. In each of the earlier cases a typical flare spray or blow-off occurs, characterized by rapid expulsion of material but small $\mathrm{H} \alpha$ brightness. Figure 1 shows such an event before the region rotated behind the limb. Figure 2 shows the time profile and spectral variation for that flare.

At 22:42:12 UT a bright eruption first appeared above the west limb and rose

Solar Physics 9 (1969) 269-277; (C) D. Reidel Publishing Company, Dordrecht-Holland

(C) Kluwer Academic Publishers - Provided by the NASA Astrophysics Data System 

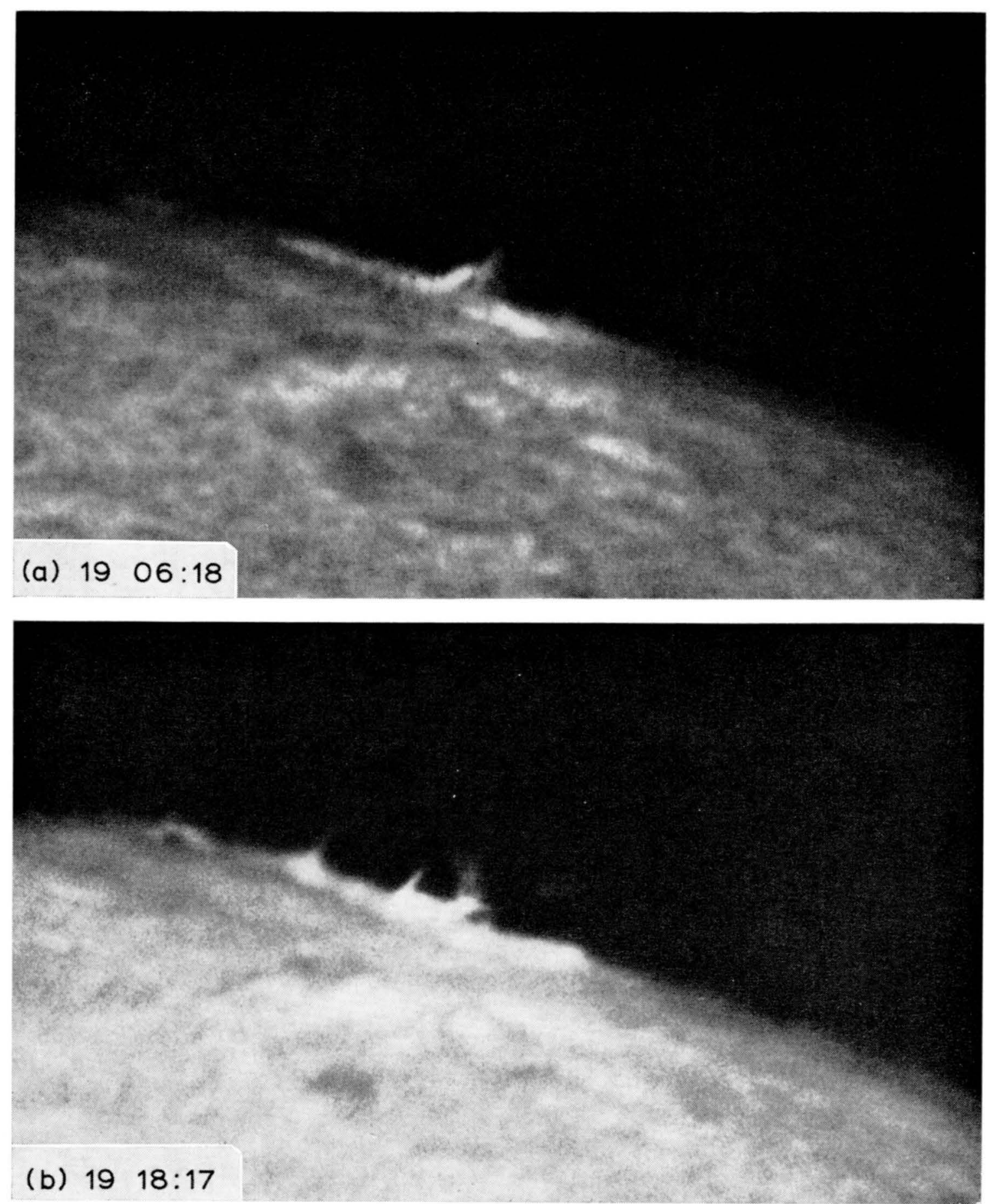

Fig. 1. Explosive spray producing X-ray burst with max at 19:06 UT. Note that the region was still on the limb at this point. 
TABLE I

X-ray data coverage, 2 December 1967

\begin{tabular}{|c|c|c|c|c|c|}
\hline \multirow{2}{*}{$\begin{array}{l}\text { Interval } \\
\text { (UT) }\end{array}$} & \multicolumn{3}{|c|}{ Bursts, $7.7-12.5 \mathrm{keV}$} & \multirow{2}{*}{$\begin{array}{l}\text { Peak Flux } \\
\text { Counts }\left(\mathrm{cm}^{2} \mathrm{sec}\right)^{-1}\end{array}$} & \multirow{2}{*}{$\begin{array}{l}\text { Background } \\
\text { Counts }\left(\mathrm{cm}^{2} \mathrm{sec}\right)^{-1}\end{array}$} \\
\hline & Begin & $\operatorname{Max}$ & End & & \\
\hline $1405-1422$ & $1405 \mathrm{E}$ & $1405 \mathrm{E}$ & $1422 \mathrm{D}$ & $56 \mathrm{D}$ & \\
\hline \multirow[t]{2}{*}{$1541-1602$} & 1544 & 1547 & 1557 & 118 & 96 \\
\hline & & 1559 & & 122 & 100 \\
\hline \multirow[t]{2}{*}{$1716-1745$} & & 1727 & & 160 & 40 \\
\hline & 1737 & 1741 & $1745 \mathrm{D}$ & 160 & 38 \\
\hline $1803-1813$ & & 1806 & & 58 & 48 \\
\hline $1852-1923$ & 1856 & 1906 & 1923D & 580 & 24 \\
\hline \multirow[t]{2}{*}{$2043-2124$} & $2043 \mathrm{E}$ & $2043 E$ & 2049 & 47D & 21 \\
\hline & & 2102 & & 34 & 24 \\
\hline \multirow[t]{2}{*}{$2203-2254$} & $2203 E$ & $2203 E$ & 2237 & $230 \mathrm{D}$ & 30 \\
\hline & 2243 & 2246 & 2253 & 950 & 28 \\
\hline \multirow[t]{5}{*}{$2339-0034$} & 2343 & 2350 & 2353D & 24 & 12 \\
\hline & & 0002 & & 42 & 14 \\
\hline & & 0008 & & 20 & 13 \\
\hline & & 0021 & & 48 & 13 \\
\hline & & 0031 & & 23 & 13 \\
\hline
\end{tabular}

1850: Growth and eruption of small loop; apparent material movement along the loop; all material disappeared back into limb by about 1920 .

2025: Growth of bright area at limb; formed small prominence which erupted about 2100 and faded out of visibility.

2140: Eruption from limb toward the south (apparently larger event than that of 2025); expands to south and fades by 2200 .

2241: Beginning of main event.

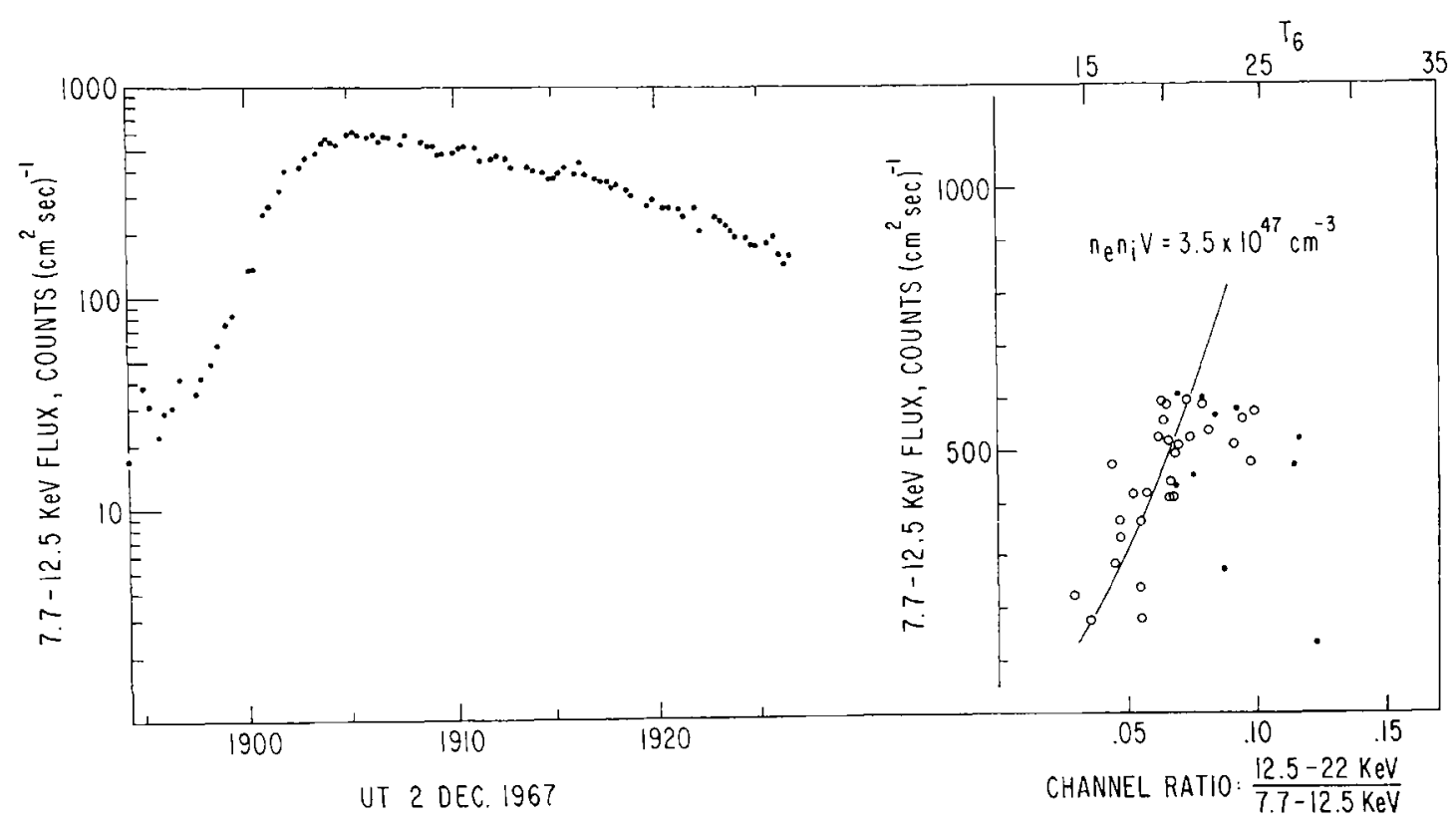

Fig. 2. Left: Time dependence of 7.7-12.5 keV flux. Right: Ratio of counts for 7.7-12.5 keV and 12.5-22 keV. The upper scale indicates the temperature corresponding to the various ratios under the bremsstrahlung hypothesis. Dots represent the rising branch; circles, the decaying branch. Note that the rising points are scattered by comparison with Figure 4. 

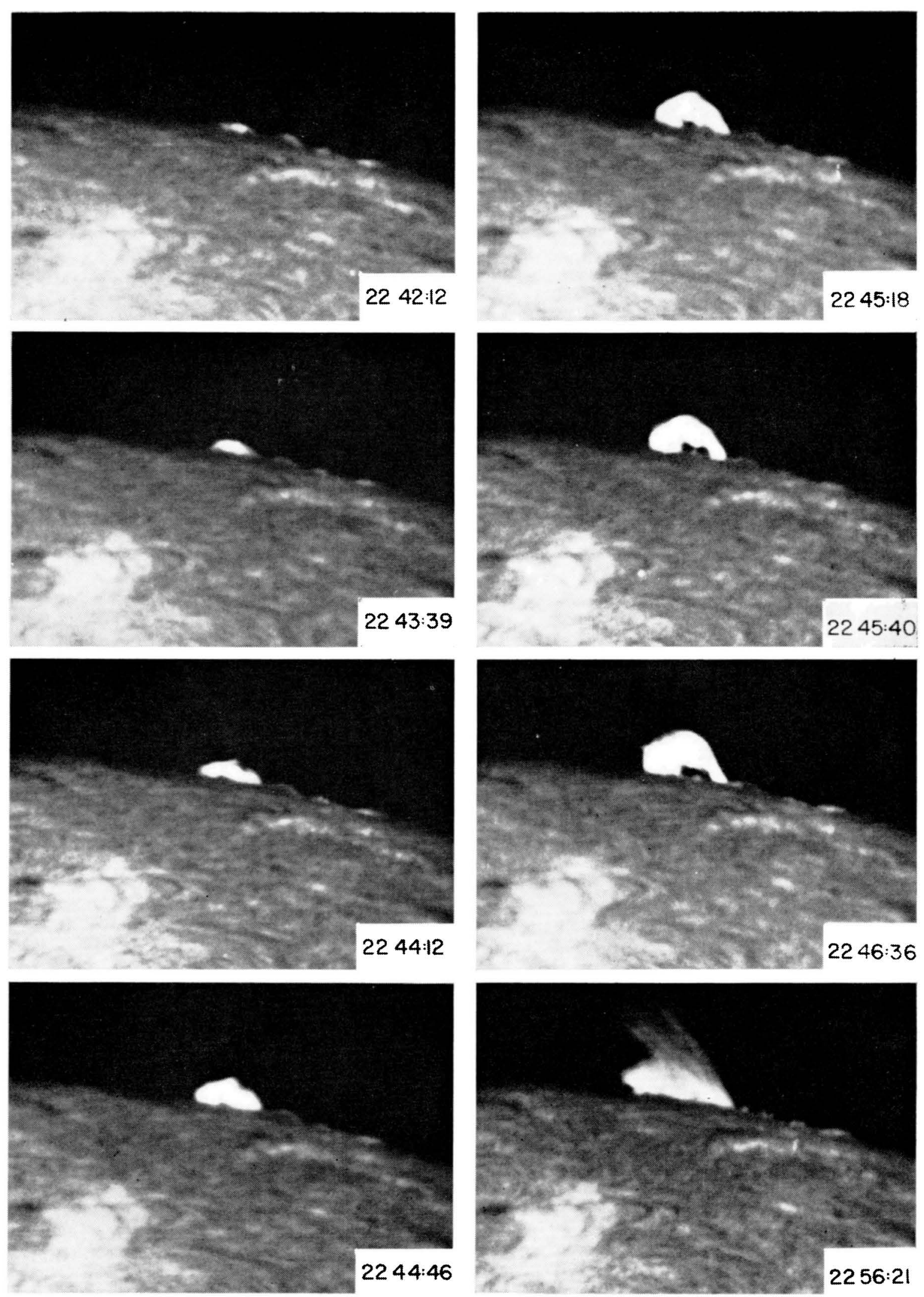

Fig. 3. Development of the bright arch on 2 December 1967 (Caltech photoheliograph). 


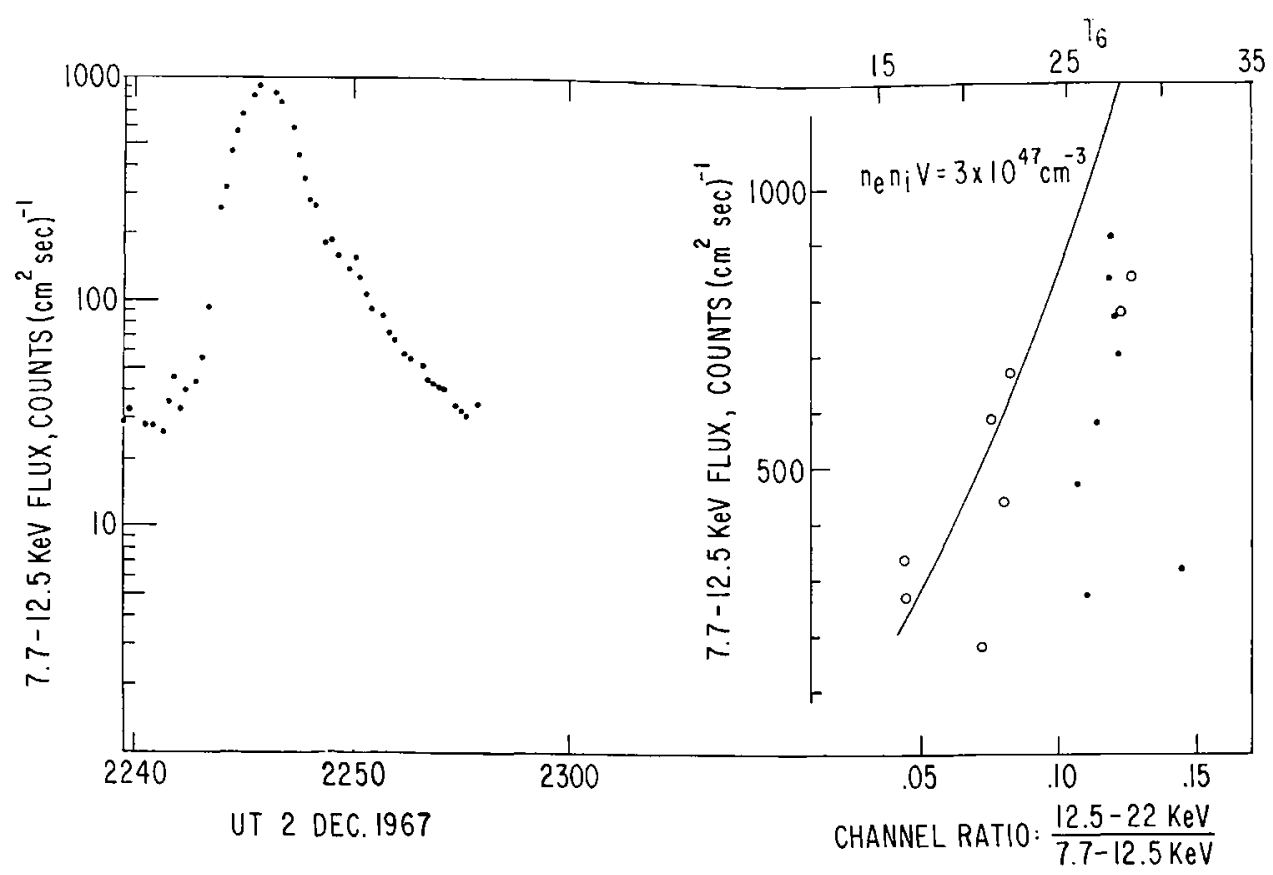

Fig. 4. Left: Time dependence of 7.7-12.5 keV flux. Right: Ratio of counts in channels for 7.7-12.5 $\mathrm{keV}$ and $12.5-22 \mathrm{keV}$. The ratios on the rising branch cluster around $0.10\left(25^{6} \mathrm{deg}\right)$, while those on the falling branch are well approximated by the line corresponding to $N_{e} N_{i}=3 \times 10^{47} \mathrm{~cm}^{-3}$, with falling temperature.

rapidly to a large arch. This eruption (Figure 3) was classed as a 2B flare by Lockheed, and appeared brighter against the sky than a normal plage on the disk. It was accompanied by an X-ray burst with an unusually steep rise and maximum flux of 950 phot $\left(\mathrm{cm}^{2} \mathrm{sec}\right)^{-1}$, about 30 times the background. Figure 4 shows the time profile of the $\mathrm{X}$-ray counting rate and the $\mathrm{X}$-ray spectral variation. Figure 5 a shows the increase of projected area with time; Figure 5b shows photon flux from $7.7-12.5 \mathrm{keV}$ vs. the projected area. The latter relation is almost exactly linear up to the X-ray maximum at 22:45:42 after which the flux dropped abruptly with an e-folding time of two minutes even through the apparent area continued to increase. When projected in cine, the prominence appears qualitatively to expand sharply after the X-ray maximum. However Figure 5a shows the rise to be uniform in time, so there is probably no such effect. Teske has kindly communicated the University of Michigan 8-12 $\AA$ data from OSO-III; he recorded a burst beginning $22: 43: 40$, max at $22: 46: 50$, end at $22: 52: 55$, with flux rising from $0.0063 \mathrm{ergs} \mathrm{cm}^{-2} \mathrm{sec}^{-1}$ to $0.0106 \mathrm{ergs} \mathrm{cm}^{-2} \mathrm{sec}^{-1}$. This compares with a typical value of $0.1 \mathrm{ergs} \mathrm{cm}^{-2} \mathrm{sec}^{-1}$ for $2 \mathrm{~B}$ flares. A $2000 \mathrm{MHz}$ radio burst was detected with a peak flux of $7 \times 10^{-22}$ watt $\left(\mathrm{M}^{2} \mathrm{~Hz}\right)^{-1}$ at $22: 46: 30$ and lasting from 22:45:00 to 22:48:00. The burst did not appear at $3750 \mathrm{MHz}$. Teske and Thomas (1969) compared the 8-12 $\AA$ flux with $2800 \mathrm{MHz}$ flux for a large number of events, and the relative $8-12 \AA$ and microwave fluxes for this event are about typical for the events they studied. The peak X-ray flux was not great enough to produce a substantial shortwave fadeout.

It is interesting that the position of the flare was actually $20000 \mathrm{~km}$ north of the 

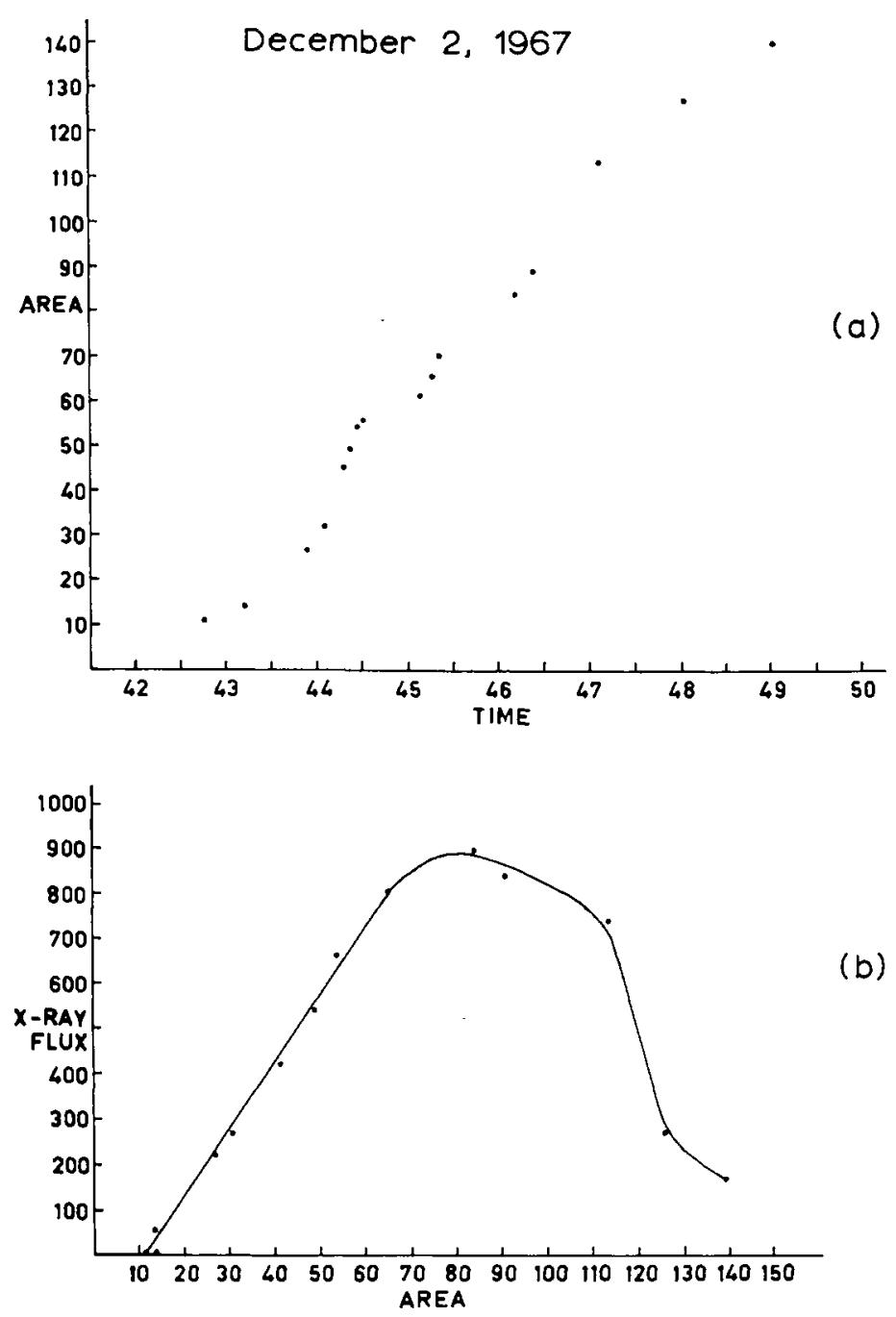

Fig. 5. a) Dependence of flare area on time. The area is plotted in arbitrary units measured on the film; $10 \mathrm{~cm}^{2}$ corresponds to $3.7 \times 10^{17} \mathrm{~cm}^{2}$ at the sun. b) Dependence of X-ray flux on area. The dependence is essentially linear up to the point of X-ray maximum.

position of the main sunspot that had passed over the limb, marked by material that remained visible in the form of small bright mounds in the chromosphere. This is not uncommon in such eruptive flares.

\section{Analysis}

We assume that the X-ray burst observed came from material in the eruptive flare visible above the solar limb. This assumption is justified by the time coincidence of area and X-ray flux increase, the absence of other observed $\mathrm{H} \alpha$ events, and the occurrence of similar coincidences in other cases (Hudson et al., 1969). The X-ray e-folding rise time of 45 seconds was considerably faster than the average of $85 \mathrm{sec}$. This suggests that the electrons had already been accelerated in the flare and were being revealed to us as the material rose above the limb.

The linearity of the X-ray flux-area relationship could be accidental since the flux 
dropped abruptly after maximum even though the area continued to increase. Energy loss or escape of the fast electrons could have caused the flux-area relationship to be linear when the de-occultation of the X-ray source was not strictly proportional to the visible area. However, it seems more reasonable to follow obvious correlations than to invent coincidences.

The linear flux rise shows clearly that there is no single kernel from which the bulk of the emission came, for in that case we should observe a step function increase in the emission. The radiating electrons must have been distributed uniformly throughout the arch.

We assume the X-rays were produced by bremsstrahlung. According to Figure 4 the flux $F$ in the $7.7-12.5 \mathrm{keV}$ channel during the rise is related to the area by the following formula

$$
F=4.7 \times 10^{-16} A \text { photons }\left(\mathrm{cm}^{2} \mathrm{sec}\right)^{-1}
$$

where $A$ is the projected area in $\mathrm{cm}^{2}$. Because of the observed linearity we assume the thickness $L$ of the flare along the line of sight was constant and equal to $15000 \mathrm{~km}$. The emission $/ \mathrm{cm}^{3}$ from the flare will be

$$
\frac{F}{A L}=\frac{4.7 \times 10^{-16}}{15 \times 10^{8}}=3.1 \times 10^{-25} \mathrm{counts} / \mathrm{cm}^{3} \mathrm{sec}
$$

which may be equated to the thermal bremsstrahlung formula (Allen, 1962), divided by $h v$ and $1 \mathrm{AU}^{2}$ and integrated over the channel energy:

$$
\begin{aligned}
& \frac{F}{A L}=3.65 \times 10^{-39} g N_{h e} N_{p} \frac{1}{T} \int_{7.7}^{12.5} \mathrm{e}^{-h v / k T} \frac{\mathrm{d} h v}{h v} \operatorname{counts}\left(\mathrm{cm}^{3} \mathrm{sec}\right)^{-1} \\
& \left(N_{p}=\text { total density of protons } ; N_{h e}=\text { density of hot electrons }\right) .
\end{aligned}
$$

Comparison of the count rates in the lowest two channels gives a 'temperature' of about $27000000 \mathrm{~K}$ or $2.3 \mathrm{keV}$. Therefore $N_{h e} N_{p}=7.1 \times 10^{19} \mathrm{~cm}^{-6}$. If we assume that there was no 'field' (cooler) material and further assume overall charge neutrality, then $N_{p}=N_{h e}$ and we find a 'hot' electron density $N_{h e}=8.4 \times 10^{9} \mathrm{~cm}^{-3}$. This gives the maximum $N_{h e}$. However, the observed $\mathrm{H} \alpha$ emission indicates that there was a certain density of cooler plasma in the arch. This density cannot be calculated directly from the $\mathrm{H} \alpha$ intensity because the details of excitation are unknown. A maximum density of field electrons is calculated below from the decay rate of the X-ray emission. The Michigan data corresponds to about $2 \times 10^{7}$ counts $/ \mathrm{cm}^{2} \mathrm{sec}$ at $8-12 \AA$. Since exponentials would only give a factor 100 difference (or $10^{5}$ counts), the extra contribution of lines is clear. We cannot admit additional cool material because of the maximum calculated below.

\section{Decay Modes}

It would be valuable to understand the time history of the production and decay of 
the energetic electrons in an X-ray burst as clues to understanding the physical conditions in the flare region. Although the energy input mechanism is unknown, the possible decay modes almost certainly are the following:

i) escape of energetic electrons from magnetic trapping

ii) coulomb collisions with cool, ambient gas

iii) radiation loss (collisional or synchrotron)

Energy loss by radiation can be shown to be too slow to account for the observede-folding decay times which ranged from about $50 \mathrm{sec}$ just after maximum to about $150 \mathrm{sec}$ near the end of the burst. Escape and collisional decay mechanisms are modeldependent and therefore are difficult to assess. It is also possible that there is appreciable energy input during the decay phase which could confuse the identification of the dominant decay mechanism.

The energy exchange time of energetic electrons of speed $V$ with electrons in a Maxwell distribution at temperature $T$ is given by Takakura and Kai (1966):

$$
T_{e e}=\frac{5 \times 10^{-32}}{N_{f}} \frac{V^{5}}{T} \mathrm{sec}
$$

where $N_{f}$ is the number of density of the cooler ambient electrons. The exchange of energy with the ion component of the field plasma is $M_{e} / M_{e}=1836$ times slower and is therefore negligible. For $N_{f}=10^{9}$ and $T=10^{6} \mathrm{~K}$ we find $T_{e e}=315 \mathrm{sec}$. The observed decay time of $50 \mathrm{sec}$ puts an upper limit of $6.3 \times 10^{9}$ electrons $/ \mathrm{cm}^{3}$ on the field electron density in the arch. Combining this with Equation (3) and requiring overall charge neutrality, we find the maximum $N_{p}$ to be $1.2 \times 10^{10} \mathrm{~cm}^{-3}$ and the minimum $N_{h e}$ to be $6 \times 10^{9} \mathrm{~cm}^{-5}$.

Even though we can determine a maximum density from the coulomb braking decay time, this mechanism seems unlikely as the cause of the actual decay, for it preferentially slows down the slower electrons and leads to a steepening of the spectrum with time. The opposite is observed. In Figure $4 \mathrm{~b}$ we see that the hardness is constant on the increasing branch (dots) while it decreases on the falling branch (circles). This is consistent with the idea that the flux increases through the de-occultation of energetic electrons already present; but is shows that decay only begins after the maximum, and affects the faster electrons preferentially. This dependence on electron speed is not consistent with coulomb braking. Therefore there must be appreciable escape from the X-ray producing region, beginning at the time of maximum. If, as is likely, the probability of escape is greater for more energetic electrons, the observed softening of the X-ray spectrum during the decay can be explained. In this connection we must recall that the electrons can traverse the flare in $1 \mathrm{sec}$ and quickly discover leaks in containment. The time of $50 \mathrm{sec}$ makes the escape difficult to describe in terms of a simple model.

The observed sharp increase in the projected area of the arch near X-ray maximum may have signalled the breaking up of suitable trapping fields in the arch. The X-ray maximum was approximately at the beginning of the large surge from one end of the arch. Solar electrons were subsequently detected at 1 AU (Lin, personal communi- 
cation), indicating that some of the electrons which had escaped trapping were able to propagate outward.

It is clear that the X-ray flux from this event is smaller than one might expect from a class $2 \mathrm{~B}$ flare. It is unlikely that this is due to the height from which the radiation comes; the November 20, 1960 flare (Zirin, 1964) produced a huge X-ray flux (judging from ionospheric data) at the same height above the photosphere. The temperature determined from the X-ray spectrum for the December 2 event is only $27000000^{\circ}$, compared with $100 \times 10^{6} \mathrm{~K}$ which is typical for $2 \mathrm{~B}$ events. It would appear that this event was overrated on the basis of its area. It was much smaller than huge eruptive events like November 20, 1960 or July 20, 1961 which did not show large, organized $\mathrm{H} \alpha$ structure but blew everything away.

\section{Conclusions}

The identity of the X-ray source with the rising arch in the initial part of the eruption is well established. The X-radiating electrons must therefore co-exist with the cooler plasma responsible for the $\mathrm{H} \alpha$ emission; the product of super-thermal electron density $N_{h e}$ and ambient density $N_{p}$ is $N_{h e} N_{p}=7.1 \times 10^{19} \mathrm{~cm}^{-6}$. The decay of the burst cannot be explained by Coulomb collisions and must be dominated by escape of the energetic electrons during the initiation of the large surge. However, the absence of coulomb decay limits the field particle density to $6 \times 10^{9}$. The relaxation of magnetic containment occurred when the X-ray temperature reached the equivalent of $\mathrm{H}=30 \mathrm{G}$ based on the density $10^{10} \mathrm{~cm}^{-3}$ at $27 \times 10^{6} \mathrm{~K}$.

The height above the photosphere on the top of the $\mathrm{X}$-ray source was approximately $20000 \mathrm{~km}$. The data do not rule out the possibility of occulted X-radiation from a still lower altitude.

\section{Acknowledgements}

This work was supported by the National Aeronautics and Space Administration under grant NASA NGR 05002 034, and the National Science Foundation under Grant 1472 (H.Z. and W.I.) and by NASA Contract NAS5-3177 (H.H. and D.M.). We wish to thank Prof. L. E. Peterson and Mr. D. A. Schwartz for encouragement and valuable suggestions.

\section{References}

Allen, C. W.: 1962, Astrophysical Quantitities, Athlone Press, London.

Hudson, H., Peterson, L. E., and Schwartz, D. A.: 1969, Astrophys. J. (to be published July 1969). Lin, R. P.: private communication.

Takakura, T. and Kai, K.: 1966, Publ. Astron. Soc. Japan 18, 60.

Teske, R. G.: 1968, private communication.

Teske, R. G. and Thomas, R. J.: 1969, submitted to Astrophys. J.

Zirin, H.: 1964, Astrophys. J. 140, 1216. 